\title{
DE L'INTÉGRATION À L'INCLUSION. UNE NOUVELLE ÉTAPE DANS L'OUVERTURE DE L'ÉCOLE AUX DIFFÉRENCES
}

Serge Thomazet

Armand Colin | « Le français aujourd'hui »

2006/1 n 152 | pages 19 à 27

ISSN 0184-7732

ISBN 9782200921071

Article disponible en ligne à l'adresse :

https://www.cairn.info/revue-le-francais-aujourd-hui-2006-1-page-19.htm

Distribution électronique Cairn.info pour Armand Colin.

(C) Armand Colin. Tous droits réservés pour tous pays.

La reproduction ou représentation de cet article, notamment par photocopie, n'est autorisée que dans les limites des conditions générales d'utilisation du site ou, le cas échéant, des conditions générales de la licence souscrite par votre établissement. Toute autre reproduction ou représentation, en tout ou partie, sous quelque forme et de quelque manière que ce soit, est interdite sauf accord préalable et écrit de l'éditeur, en dehors des cas prévus par la législation en vigueur en France. Il est précisé que son stockage dans une base de données est également interdit. 


\title{
DE L'INTÉGRATION À L'INCLUSION
}

\section{Une nouvelle étape dans l'ouverture de l'école aux différences}

\author{
Par Serge THOMAZET \\ Université du Québec à Chicoutimi \\ Laboratoire PAEDI (JE 2432) à Clermont-Ferrand
}

"Plus qu'un simple instrument de communication, le langage illustre la façon dont on se représente mentalement une réalité.

Il n'est donc pas étonnant que les mots employés pour parler des personnes handicapées aient fait l'objet d'une remise en question parallèle à l'évolution de leur place dans la société. "

Patrick Fougeyrollas (repris par Hamonet, 2002)

\section{Introduction}

L'intégration scolaire est un sujet important de débats et de recherches. Le terme inclusion, utilisé principalement dans les pays anglophones, est récemment venu concurrencer, dans de nombreux pays dont la France, le terme intégration pour désigner les pratiques de scolarisation « au plus près de l'école ordinaire".

Aux États-Unis, même si le terme inclusion (inclusion, inclusive school, inclusive education) est quelquefois synonyme d'intégration (voir par exemple Whitworth, 1999 ; Dyson, 2002), il représente le plus souvent un changement conceptuel radical pour la plupart des acteurs et chercheurs en éducation. En effet, l'école ordinaire, pour devenir inclusive, doit s'organiser pour répondre aux besoins éducatifs particuliers ${ }^{1}$ de tous les élèves alors que l'intégration, appelée mainstream aux USA (litt. : dans le courant principal) suppose la mise en œuvre de dispositifs de soutien et de rééducation pour adapter l'enfant ou l'adolescent ${ }^{2}$ à l'école ordinaire (il en est retiré lorsqu'il n'est pas jugé capable de bénéficier de l'enseignement dispensé).

En France, l'arrivée de ce terme conduit à des positions très contrastées qui vont d'un intérêt du fait de l'apriori positif envers la nouveauté en pédagogie, notamment si celle-ci vient d'outre Atlantique, au rejet sans

1. Nous appelons "besoins éducatifs particuliers" (special educational needs), les besoins d'élèves qui, lorsque les pratiques d'intégration scolaire ne sont pas mises en place, les conduisent vers des dispositifs ségrégatifs (Whitworth, 1999). Ce terme rassemble donc les élèves ayant besoin d'une adaptation de l'enseignement du fait d'une déficience (motrice, sensorielle), d'un trouble ou d'un retard mental mais aussi plus généralement tous les élèves qui du fait de leurs difficultés, peuvent se trouver exclus des parcours ordinaires de scolarisation. 2. Le masculin est utilisé à titre épicène. 
appel d'un terme que l'on imagine s'imposer en solution miracle et remettre en question les expertises et la réflexion déjà présentes.

Au moment où se met en place la loi du 11 février 2005 "Pour l'égalité des droits et des chances, la participation et la citoyenneté des personnes handicapées ", il nous semble pertinent de profiter de l'expérience des pays qui ont fait le choix de promouvoir une école inclusive pour réfléchir à la place des élèves à besoins éducatifs particuliers dans notre système éducatif. En effet, de nombreux jeunes sont encore exclus de l'école, notamment des établissements secondaires. Atteints, entre autres, d'un handicap mental, de troubles de la communication, ou en grande difficulté scolaire ou comportementale, ils n'ont pas toutes les «qualités " pour fréquenter l'école telle qu'elle est. Si, conformément au souhait de la nouvelle loi et même si celleci n'utilise pas le terme d'école inclusive, l'école publique doit être celle de tous les enfants et adolescents, il nous faut trouver des solutions pour adapter nos établissements aux besoins des élèves "différents ". C'est dans ce but que certains ont cherché à créer une école « inclusive " plutôt que de tenter à postériori d'intégrer les élèves à besoins éducatifs particuliers.

\section{Quelles sont les caractéristiques d'une école inclusive ?}

Après une période ségrégative, puis une période intégrative ${ }^{3}$, progressivement, s'est développée sous le terme d'inclusion une conception de la scolarisation au plus près de l'école ordinaire, qui suppose non seulement l'intégration physique (l'établissement spécialisé se déplace dans l'école) et sociale (les élèves à besoins particuliers partagent les récréations, repas, ateliers récréatifs... des élèves des filières régulières), mais aussi pédagogique afin de permettre à tous les élèves d'apprendre dans une classe correspondant à leur âge ceci quel que soit leur niveau scolaire ${ }^{4}$.

"L'éducation inclusive se préoccupe de tous les enfants, en portant un intérêt spécial à ceux qui traditionnellement n'ont pas d'opportunité éducative comme les enfants à besoins particuliers, avec incapacités, ou appartenant à des minorités ethniques ou linguistiques, entre autres » (UNESCO, 2001).

De nombreux travaux ont été menés dans le but de repérer les caractéristiques d'une école inclusive dans un système éducatif occidental (Skrtic et al., 1981 ; Sailor, 1991 ; Harvey, 1995 ; Ainscow, 1996 ; McLeskey \& Waldron, 1996 ; Clark et al., 1999 ; Armstrong, 2001 ; Vienneau, 2002). Sans être consensuelles, ces études convergent sur des principes directeurs et des caractéristiques que nous exposons ci-après.

Le principe fondamental est que l'école ordinaire doit accueillir tous les jeunes en s'adaptant aux besoins de chacun, tout en apportant ses réponses d'une façon qui soit la plus ordinaire possible.

3. Pour un historique des différentes étapes (dont les caractéristiques principales sont synthétisées dans le tableau ci-après) le lecteur peut se reporter à l'article de Philippe Mazereau, dans ce même numéro.

4. Pour une analyse des différentes formes d'intégration : physique, sociale et pédagogique, voir Wolfensberger \& Thomas (1983). 
L'école inclusive fait de l'établissement scolaire local le lieu d'éducation de tous les enfants ou adolescents. L'école a une politique d'acceptation qui fait qu'aucun élève n'est refusé pour la nature ou l'étendue de ses besoins particuliers. Ceux-ci ne sont pas «intégrés » dans l'école inclusive, ils y ont leur place, de plein droit, comme tous les autres élèves. L'inscription dans un établissement scolaire se fait principalement sur la base de l'âge. Chaque enfant ou adolescent fréquente donc l'école de son quartier ou de son village. Ainsi, dans chaque établissement, les élèves à besoins éducatifs particuliers sont présents dans une proportion qui ne dépasse pas celle habituellement rencontrée dans la population du quartier.

L'école inclusive répond au mieux aux besoins de chaque élève. Les besoins particuliers des élèves doivent être effectivement pris en compte, même en intégration. Chaque enseignant de classe ordinaire doit pouvoir recevoir l'aide des personnes ressources expertes en fonction des besoins particuliers de ses élèves. Ceux présentant des besoins importants qui nécessitaient, lorsqu'ils étaient en établissement spécialisés, la présence de professionnels du secteur santé tels que orthophonistes, psychologues, rééducateur... doivent pouvoir bénéficier de leur aide dans l'école ordinaire. Un projet individuel ${ }^{5}$ est élaboré par tous les partenaires concernés, chacun est conscient de la nécessité de faire les concessions nécessaires à la réalisation d'un projet harmonieux du point de vue de l'élève. Les solutions trouvées font l'objet de projets intégrant des objectifs mesurables dans un délai raisonnable. Une vigilance au quotidien permet de s'assurer de l'adéquation des dispositifs aux besoins, tandis que des révisions programmées régulièrement permettent, si nécessaire, la réactualisation du projet.

Les dispositifs spéciaux (classes spéciales, groupes d'aides, unités d'intégration) sont mis en place en réponse aux besoins des élèves effectivement présents dans l'établissement.

L'école inclusive est un processus qui amène l'établissement scolaire à trouver des solutions pour scolariser tous les enfants de la manière la plus ordinaire possible. Le fonctionnement par classe n'est qu'un aspect du fonctionnement de l'établissement qui s'est transformé pour répondre aux besoins de tous les élèves (groupes de projets, etc.). Il est notamment possible de s'appuyer sur l'organisation en cycle, le projet d'établissement et la mise en place d'une pédagogie différenciée (et non de réponses individualisées) pour organiser des ateliers dont certains peuvent être très spécialisés (hyper-stimulation, ergothérapie, cours de locomotion, langue des signes...) tout en étant intégrés dans le fonctionnement ordinaire de l'établissement ${ }^{6}$.

5. Ce type de projet existe dans tous les pays sous des noms différents (Individual Education Plan aux USA, plan d'intervention personnalisé au Québec, projet personnalisé de scolarisation en France, etc.).

6. Là encore, le principe de normalisation a été, au moins aux États-Unis, une source d'inspiration déterminante pour la définition de ces pratiques. Initialement utilisé dans les pays scandinaves pour les personnes handicapés (Lemay, 1996), il a été étendu par Wolfensberger pour toutes les personnes susceptibles de subir un désavantage (Wolfensberger, 1972; Wolfensberger \& Thomas, 1983). 
Toute pratique est pensée à priori pour être adaptée au plus grand nombre. Dans cette optique, une pédagogie de projet ou le travail à partir de situations complexes, permettent une différenciation effective sans surcharger le professeur par la gestion de groupes multiples. Le travail en partenariat au sein de l'équipe pédagogique et en liaison avec des experts permet de disposer d'un éventail de solutions qui ne se limite pas à ce que l'enseignant isolé peut mettre en place dans sa classe. Les professionnels de l'école sont engagés dans un dispositif de formation d'équipe qui leur permet de maintenir leurs connaissances et compétences à jour.

Tableau synthèse : trois modèles de prise en charge des enfants à besoins éducatifs particuliers

\begin{tabular}{|c|c|c|c|}
\hline & Ségrégation & Intégration - Mainstream & Intégration - Inclusion \\
\hline Période & $\begin{array}{l}\text { Première conception, présente depuis } \\
\text { les débuts de l'éducation spéciale }\end{array}$ & $\begin{array}{l}\text { Ancienne conception de l'intégration, } \\
\text { depuis } 1968 \text { en Catalogne*, } 1975 \text { aux USA } \\
\text { et en France, } 1978 \text { au Québec }\end{array}$ & $\begin{array}{l}\text { Nouvelle conception, apparue vers } 1988 \\
\text { en Catalogne et en } 1986 \text { aux USA. } \\
\text { Discutée sous des termes différents } \\
\text { (intégration...) dans les autres pays }\end{array}$ \\
\hline Système éducatif & Ségrégatif & Intégratif (physique ou social) basé sur la sélection & $\begin{array}{l}\text { Intégratif (pédagogique) } \\
\text { et compréhensif (englobant) }\end{array}$ \\
\hline $\begin{array}{l}\text { Quelles adaptations } \\
\text { pour les enfants à besoins } \\
\text { éducatifs particuliers? }\end{array}$ & $\begin{array}{l}\text { Les enfants à besoins éducatifs particuliers } \\
\text { peuvent recevoir une instruction au sein } \\
\text { d'établissements prenant en charge } \\
\text { prioritairement leurs besoins médicaux } \\
\text { et éducatifs. }\end{array}$ & $\begin{array}{l}\text { Scolarisation au plus près d'une école ordinaire } \\
\text { auquel l'enfant doit s'adapter. Plus les besoins des } \\
\text { enfants sont importants, plus les e détours ségrégatiffs ” } \\
\text { (aide, classes spéciales, cours ou moments de soutien) } \\
\text { le sont aussi. }\end{array}$ & $\begin{array}{l}\text { Les pratiques spécialisées sont « normalisées *, } \\
\text { elles s'insèrent dans les dispositiffs ordinaires } \\
\text { de différenciation de la classe et de l'école } \\
\text { (pédagogie différenciée). }\end{array}$ \\
\hline $\begin{array}{l}\text { Contraintes } \\
\text { institutionnelles }\end{array}$ & $\begin{array}{l}\text { Scolarisation dans des structures } \\
\text { particulières }\end{array}$ & Incitation à intégrer (lorsque cela est possible...) & Obligation scolaire pour tous \\
\hline $\begin{array}{l}\text { Rôle des professionnels } \\
\text { de l'éducation spéciale }\end{array}$ & \multicolumn{2}{|c|}{ Aide aux enfants, qui reçoivent un enseignement spécial } & $\begin{array}{l}\text { Aide aux personnels de l'école } \\
\text { (qui doivent adapter leurs interventions) }\end{array}$ \\
\hline Modèle pédagogique dominant & \multicolumn{2}{|c|}{$\begin{array}{l}\text { Pédagogie thérapeutique : les enfants ont besoin d'une pédagogie spéciale } \\
\text { basée sur leur déficience, à visée réparatrice. }\end{array}$} & $\begin{array}{l}\text { Pédagogie différenciée : les enfants reçoivent } \\
\text { un enseignement différencié en fonction } \\
\text { de leurs besoins }\end{array}$ \\
\hline Modèle d'intégration & $\begin{array}{l}\text { \| n'est pas pertinent de mettre à l'école } \\
\text { ordinaire des enfants qui ont des besoins } \\
\text { « extraordinaires " }\end{array}$ & Médical et assisté & Cognitif et humaniste \\
\hline $\begin{array}{l}\text { Exemple d'évolution } \\
\text { des terminologies }\end{array}$ & $\begin{array}{l}\text { Handicapé } \\
\text { Classe spéciale }\end{array}$ & $\begin{array}{l}\text { Enfant handicapé } \\
\text { Classe visant l'intégration (ex : CLIS) }\end{array}$ & $\begin{array}{l}\text { Enfant en situation de handicap } \\
\text { Élève à besoins éducatifs particuliers } \\
\text { Unité d'intégration }\end{array}$ \\
\hline
\end{tabular}

* Pour la situation catalane, voir Jiménez (2003)

\section{L'évolution de l'intégration à l'inclusion résulte d'une évolution des conceptions sur le handicap}

Depuis plusieurs décennies l'éducation des élèves désignés aujourd'hui comme ayant des besoins éducatifs particuliers n'a jamais dévié de son objectif : apporter la meilleure éducation possible afin de faciliter leur intégration dans la société. Ainsi, en France, la fréquentation des établissements spécialisés était-elle pensée comme un " détour ségrégatif " destiné à faciliter la future intégration sociale de ces élèves (Mège-Courteix, 1999). Sans méconnaitre les visées implicites de relégation des filières d'éducation spécialisée au début du $20^{\mathrm{e}}$ siècle (Gateaux, 1999), il nous semble que ce mode d'organisation correspond avant tout aux modes de pensée de l'époque concernant l'enfant, l'éducation et la différence. Modes de pensée marqués par des valeurs culturelles et sociales qui ont conduit à envisager un cadre ségrégatif puis ordinaire pour l'éducation des personnes à besoins éducatifs particuliers. Les discussions actuelles sur les pratiques inclusives n'étant qu'une nouvelle déclinaison de l'objectif d'intégration sociale. 


\section{Une conception du handicap en termes de désavantage plutôt que de déficience.}

Une source déterminante de changement dans la façon de penser la scolarisation des élèves à besoins éducatifs particuliers vient de notre perception du handicap. En effet, à partir de la fin des années 1960 les acteurs du secteur (médecins, personnels paramédicaux) prennent conscience du fait que les états handicapants sont beaucoup plus répandus, variés et complexes que ne le donnent à penser les classifications (Ainscow, 1996). Les classifications elles-mêmes évoluent rapidement s'éloignant d'une catégorisation objective et immuable de faits biologiques (paralysie, cécité...) pour prendre en compte la relation des personnes à la vie sociale. Ainsi, prend-on conscience que le "normal " «implique une conception de la société qui l'assimile subrepticement et abusivement à un système de déterminismes, alors qu'elle est un système de contraintes " (Canguilhem, 1966).

Ces changements traduisent une évolution des points de vue sur le handicap passant d'un modèle médical à un modèle social (Fougeyrollas, 1995 ; Armstrong, 2003). Ils sont aussi révélateurs des difficultés d'un dispositif avant tout orienté vers l'action (allocations, placements). Les ambigüités des classifications conduisant à multiplier les critères d'appartenance aux catégories autant que les catégories elles-mêmes (voir par exemple l'analyse de Maurice, 1995 dans le cas du retard mental). Bref, plus on travaille le concept de handicap, moins on sait ce qu'est une personne handicapée, le concept devient polysémique (Salbreux, 2002) connoté négativement, inopérant pour la recherche et l'on prend conscience qu'il est avant tout une construction sociale (Plaisance, 1999); le degré de handicap étant fonction de la compensation sociale que la personne reçoit.

L'organisation mondiale de la santé (OMS) a entériné ces évolutions dans ses révisions de la classification internationale du handicap en 1980 puis en 2002 (cf. Hamonet, 2002, pour un historique). Ces évolutions ont amené à des changements de dénomination particulièrement intéressants : de handicapé, on est passé à personne handicapée puis à personne en situation de handicap. Ces changements de termes sont révélateurs des trois étapes par lesquelles sont passés les modèles du handicap d'abord centrés sur la maladie (déficience), puis sur la personne (incapacité) et enfin sur la situation (désavantage). Ces qualifications du handicap proposées par Philip Wood dans le cadre de ses travaux pour l'OMS s'avèrent déterminantes pour comprendre l'évolution de l'attitude de nos sociétés envers la différence (voir par exemple Maudinet, 1999).

Ainsi, de nos jours il est admis que les conséquences du handicap sont, du moins pour l'école, plus importantes que le handicap lui-même (le concept opérant étant alors désavantage plutôt que déficience). De plus, cette évolution vers une conception sociale du handicap amène à prendre en considération de nombreuses situations où des personnes subissent un

7. Typologies des handicaps permettant de déterminer qui est handicapé, à quel degré, etc. Ces typologies sont présentées dans l'article de Catherine Dorison, dans ce même numéro. 
désavantage sans qu'il ait pour origine une maladie ou une déficience (Fougeyrollas, 1995).

La conception du handicap a donc changé, on le perçoit maintenant en terme de désavantage. En conséquence c’est la société, et dans le cas de l'école, l'établissement scolaire et les prises en charge éducatives offertes aux élèves qui conduisent, ou non, à des situations handicapantes pour les élèves porteurs d'une déficience, et plus généralement pour tout élève à besoins éducatifs particuliers. De fait, on s'aperçoit que l'approche inclusive ne désigne pas simplement une intégration "poussée ", elle résulte d'un changement paradigmatique et conduit à un changement structurel (Harvey, 1995) de l'école qui a maintenant la mission d'accompagner tous et chaque enfant à avancer au mieux dans les apprentissages.

\section{Inclusion : quelle pertinence pour le système français ?}

Dans le cas de la France, le terme en usage - intégration - utilisé depuis plus de 20 ans, regroupe des réalités très différentes allant de l'intégration-mainstream à l'école inclusive. La conséquence de cette polysémie est que parler d'intégration ne nous informe pas sur les pratiques effectives. Par exemple, le fait de dire d'un enfant qu'il suit une scolarité en intégration scolaire veut-il dire qu'il fréquente une classe spécialisée dans une école ordinaire (intégration-mainstream) ou que sa scolarité se fait en classe ordinaire, avec le soutien d'une unité d'intégration (ce qui se rapprocherait de l'école inclusive) ?

Repérer dans les attitudes et pratiques, celles qui relèvent d'une école inclusive aurait le mérite de la clarté. Par ailleurs, leur promotion nous semble offrir plusieurs opportunités au système éducatif français :

L'opportunité d'une évolution structurelle de l'école. L'intégration s'est mise en place dans une école dont une fonction était de repérer des élites. Dans cette école, une fois les bases du lire/écrire/compter acquises, il n'y avait aucun sens, pas plus économique que social à ce que tous les enfants réussissent (Baudelot \& Establet, 1971). C'est cette école, avec une organisation pratiquement semblable, qui doit maintenant faire en sorte que tous les enfants qui lui sont confiés réussissent au mieux de leurs possibilités (Marshak, 2003). Il n'est pas étonnant alors que l'intégration ne se développe pas vraiment depuis 20 ans, confrontée à une impossibilité structurelle. Par contre, l'inclusion, que l'on pourrait considérer comme une réponse spécifique à une population limitée, interroge bien au-delà "les visées, les pratiques et les stratégies de toute entreprise éducative " (Gardou \& Develay, 2001).

Par le développement de pratiques inclusives, on pourrait ainsi espérer dépasser le dilemme dans lequel se trouvent de nombreux enseignants confrontés à des publics hétérogènes : enseigner aux meilleurs, au risque de perdre les plus faibles ou enseigner aux plus faibles, au risque de faire baisser le niveau, à moins que l'on enseigne à "l'élève moyen standard ", ralliant ainsi la majorité, tout en délaissant les marges! Par une transformation en profondeur de l'école, l'approche inclusive a la prétention de rejoindre tous les élèves, sans faire baisser le niveau, autrement dit, d'être véritablement équitable. 


\section{Nous pourrions espérer dépasser la stagnation constatée dans de nombreux pays occidentaux de l'accueil à l'école ordinaire des enfants et adolescents à besoins éducatifs particuliers (voir, par exemple} Zucman, 2002, pour la France). En effet, si l'on considère que l'intégration des enfants qui pouvaient l'être sans adaptation autre que matérielle est déjà bien avancée (c'est le cas notamment de la plupart des enfants souffrant d'un handicap sensoriel ou moteur). L'étape suivante consiste à intégrer des élèves qui n'ont pas accès aux apprentissages dans le cadre d'un enseignement traditionnel. En France, plus de 90000 enfants et jeunes sont en attente d'une scolarité ordinaire (CTNERHI DREES et DGAS, 2004) du fait d'un handicap, et beaucoup plus encore si l'on prend en compte les élèves en grande difficulté scolaire et/ou comportementale.

L'opportunité d'aider tous les élèves qui en ont besoin. Si la réflexion menée concerne bien souvent les élèves présentant des déficiences importantes, elle constitue une opportunité pour aider les élèves en difficulté " ordinaire", et pour lesquels les moyens sont souvent limités. Ainsi, apporter une réponse aux besoins « éducatifs » de chaque enfant et adolescent, sans nécessairement opérer une classification médicale ou sociale rend possible, par exemple, la mise en place d'un groupe d'aide en lecture pour les élèves qui en ont besoin, ceci quelle que soit l'origine de leurs difficultés ou de leurs déficiences (Thomazet, 2000). De plus, cette approche relance un questionnement sur la pertinence de certaines filières telles que les sections d'enseignement général et professionnel adapté (SEGPA) au recrutement sociologiquement marqué (Goigoux, 1999) alors que les moyens dont ils disposent pourraient constituer une aide efficace pour tous les élèves en difficulté des collèges.

Une école réellement inclusive, on le voit encore ici, est dépendante de la capacité de l'école - et donc de ses enseignants à mettre en place une différentiation pédagogique - et à être innovants. Croire qu'une démocratisation de l'école consiste à donner à tous l'enseignement qui a jusqu'ici convenu à un petit nombre de privilégiés autrefois, c'est condamner des élèves à affronter des contenus et des modes d'enseignement auxquels, pour des raisons personnelles, sociales ou familiales, ils ne sont pas préparés.

La nouvelle loi pour l'égalité des droits et des chances apporte des réformes intéressantes et son application offre la possibilité d'avancées significatives vers l'école inclusive.

L'établissement scolaire du secteur, devient l'école de chaque enfant. On passe de la "préférence " (pour une scolarité ordinaire) (précédente loi de 1975) au principe général et ordinaire d'obligation scolaire. De même, la nouvelle loi a fait disparaitre toute référence à "l'éducation spéciale ", les dispositifs spécialisés devenant des ressources permettant, en fonction des besoins, l'aménagement de " parcours de formation adapté ». Enfin, même si elle continue à parler de personnes handicapées (probablement pour des raisons financières), plutôt que de personnes en situation de handicap, la loi de 2005 se situe clairement dans une approche sociale, présentant le handicap comme situationnel.

Ces aspects de la loi constituent des avancées majeures en direction de l'école inclusive. Cependant, il en est d'autres qui peuvent inquiéter, 
comme la création d'un droit à compensation, qui pourrait amener à rechercher des aides individuelles à titre de compensation du handicap, au détriment d'une réponse globale du système éducatif aux besoins de tous les élèves (Daubannay, 2005). Les USA sont largement engagés dans cette voie et chaque établissement scolaire doit faire la preuve qu'il répond aux besoins particuliers de chaque élève qu'il accueille, au risque d'avoir à financer sur ses fonds propres une scolarité mieux adaptée dans un autre établissement (Horn \& Tynan, 2001).

\section{Conclusion}

Partant d'une question d'évolution terminologique, nous avons pointé une différence conceptuelle ayant des conséquences importantes sur les pratiques éducatives pour tous les enfants. Nous avons fait remarquer qu'il pouvait y avoir des avantages, dans la situation française, à utiliser un terme pour qualifier les dispositifs éducatifs répondant aux besoins particuliers des élèves selon les principes de l'école inclusive.

Un mot est nécessaire pour désigner ces pratiques, que ce soit inclusion, ou tout autre mot que l'on jugera une traduction plus pertinente. Bien sûr, le seul changement de mot ne permettra pas de changer les pratiques, mais il permettra de penser autrement l'accueil de tous les enfants à l'école ordinaire... car les mots aussi font les choses (Plaisance, 1999).

La mise en place d'une école inclusive nécessite de relever de nombreux défis, aussi bien au niveau des attitudes, des ressources que des savoir-faire (Vienneau, 2002). Cependant, face à l'espoir quelle suscite pour de très nombreux jeunes et leurs familles, nous ne pouvons plus attendre pour " faire que l'École de tous et de chacun, l'École de tous les âges de la formation scolaire et professionnelle devienne réellement ce premier et dernier "lieu commun" des apprentissages, du partage et de la citoyenneté " (Daubannay, 2002).

\section{Serge THOMAZET}

\section{Bibliographie partielle}

(la bibliographie complète est consultable sur mon site :

http://www. auvergne.iufm.fr/ER/sthomazet/sthomazet.htm)

- Loi du 11 février 2005 pour l'égalité des droits et des chances, la participation et la citoyenneté des personnes handicapées (2005), J. O. n 36 du 12 février 2005, p. 2353 : NOR: SANX0300217L.

- PlaisanCe É. (1999), "L'éducation spéciale... ou comment les mots pour les choses ", Éducation, 17 (1), p. 49-61.

- Wolfensberger W. \& ThOmas S. (1983), Passing : programme d'analyse des systèmes de services : application des buts de la valorisation des rôles sociaux: manuel des critères et des mesures de la valorisation des rôles sociaux, Gatineau, Les Communications Opell (rééd. 1988). 


\section{Encart : au risque d'une caricature, nous tentons de décrire ce que pourrait être la scolarité au collège d'un adolescent présentant un retard mental important.}

Jean-Paul, enfant porteur de trisomie 21, est âgé de 13 ans, son efficience scolaire est très faible (il ne sait pas encore lire), mais, comme tous les adolescents de son âge, il fréquente l'école de son quartier. Son arrivée au collège a été anticipée et l'équipe éducative, en partenariat avec le dispositif spécialisé qui a suivi l'enfant à l'école primaire $\left(\mathrm{SESSAD}^{8}\right.$ et enseignant spécialisé), a pu préparer un projet individualisé. Une évaluation des habiletés sociales a permis de faciliter son entrée dans la vie du collège alors qu'une évaluation pédagogique a formalisé des objectifs à atteindre avec une échéance raisonnable, compte tenu des capacités d'apprentissages de Jean-Paul.

Jean-Paul est scolarisé la majeure partie de son temps dans une classe de sixième. Il participe à plein temps aux cours de musique, EPS, arts plastiques et, en fonction des projets mis en place par les professeurs, à certains autres cours. Il va alors participer aux mêmes activités que les autres élèves, sans pour autant avoir les mêmes objectifs (par exemple, il peut travailler en biologie sur la croissance des plantes avec des élèves de $6^{\mathrm{e}}$ qui eux, vont travailler sur la notion d'espèces). À certains moments (au moins deux heures par jour), l'organisation des cours (travaux de groupes, ateliers) permet de mettre en place les apprentissages particuliers nécessaires à Jean-Paul (et à d'autres enfants certainement).

Par ailleurs, Jean Paul a des besoins non scolaires (orthophonie, psychomotricité, développement d'une vie sociale autonome) qui pourraient prendre place pendant le temps scolaire afin de ne pas surcharger son emploi du temps. La réponse à ces besoins nécessite des moyens. Certains sont déjà disponibles dans l'établissement (professeurs), d'autres peuvent être apportés par le SESSAD, d'autres enfin doivent être ajoutés (par exemple, intervention d'enseignants spécialisés pour un travail de coordination et d'intervention pédagogique, par exemple pour l'apprentissage de la lecture).

8. Service d'éducation spécialisée et de soins à domicile. Un SESSAD est un dispositif médicoéducatif mobile qui peut intervenir dans une école ordinaire. 